\title{
Predictores de depresión mayor y generación de un score para su screening durante la pandemia por SARS-CoV-2: subanálisis de la encuesta ImPPaCTS-SAC.20
}

\author{
Predictors of major depression and generation of a screening score in the SARS-CoV-2 \\ pandemic: Subanalysis of the survey ImPPaCTS-SAC.20 \\ Luciano Battioni', Cristhian E. Scatularo1*, Sebastián Bellia², Sara Costa de Robert², \\ Ma. de las Nieves Gatti , Mario Racki², Guillermina Soracio ${ }^{1}$, Adrián Lescano ${ }^{1}$, Julio Giorgini y \\ Stella M. Pereiro ${ }^{1}$ \\ ${ }^{1}$ Consejo de Insuficiencia Cardiaca e Hipertensión Pulmonar; ${ }^{2}$ Consejo de Aspectos Psicosociales. Sociedad Argentina de Cardiología, Ciudad \\ Autónoma de Buenos Aires, Argentina
}

\section{Resumen}

Objetivos: Describir predictores de depresión en trabajadores de la salud de Argentina durante la pandemia causada por el coronavirus 2 del síndrome respiratorio agudo grave (SARS-CoV-2) y generar un puntaje para su pesquisa. Métodos: Subanálisis de la encuesta argentina ImPPaCTS-SAC.20, incluyendo trabajadores de la salud con depresión mayor según el PHQ 9 (Patient Health Questionnaire 9). Se evaluaron predictores mediante análisis multivariante, se construyó un nuevo puntaje en la cohorte de derivación y se determinó su punto de corte, cuya calidad fue evaluada en la cohorte total y en la de validación. Resultados: Doscientos setenta trabajadores (22.1\%) presentaban depresión mayor. Los predictores de depresión mayor fueron sexo femenino $(p=0.002)$, sentirse discriminado $(p=0.001)$, uso de ansiolíticos $(p=0.001)$ y tabaquismo ( $p=0.025)$. Se elaboró un puntaje con dichas variables (sexo femenino 2 puntos, sentirse discriminado 1.5 puntos, uso de ansiolíticos 2.5 puntos y tabaquismo 3 puntos), con un área bajo la curva de 0.65 y un punto de corte de 2. En la cohorte de validación el área bajo la curva fue 0.66. Comparado con el PHQ 9 el valor predictivo negativo fue del $84 \%$. Conclusiones: Los trabajadores de la salud en Argentina presentaron elevada prevalencia de depresión mayor durante la pandemia, asociado a factores predictores. Se elaboró un puntaje para detectar a quienes tienen bajo riesgo de padecerla.

Palabras clave: Depresión. Trabajadores de la salud. SARS-CoV-2.

\begin{abstract}
Objectives: to describe predictors of depression in health workers in Argentina during SARS-CoV-2 pandemic and to generate a score for their screening. Methods: Subanalysis of the Argentine ImPPaCTS-SAC.20 Survey, including health workers with major depression according to PHQ 9 (Patient Health Questionnaire 9). Predictors were evaluated by multivariate analysis; a new score was constructed in the referral cohort and its cut-off point was determined. whose quality was evaluated in the total cohort and in the validation cohort. Results: 270 workers (22.1\%) had major depression. Predictors of major depression were female gender $(p=0.002)$, feeling discriminated against $(p=0.001)$, use of anxiolytics $(p=0.001)$ and smoking
\end{abstract}

Correspondencia:

${ }^{*}$ Cristhian E. Scatularo

E-mail: emmanuelscatularo@gmail.com
Fecha de recepción: 27-08-2021

Fecha de aceptación: 19-11-2021 DOI: $10.24875 /$ ACM.21000276
Disponible en internet: 02-02-2022 Arch Cardiol Mex. (ahead of print) www.archivoscardiologia.com 1405-9940 / ๔ 2021 Instituto Nacional de Cardiología Ignacio Chávez. Publicado por Permanyer. Este es un artículo open access bajo la licencia CC BY-NC-ND (http://creativecommons.org/licenses/by-nc-nd/4.0/). 
$(p=0.025)$. A score was prepared with these variables (female gender 2 points, feeling discriminated against 1.5 points, use of anxiolytics 2.5 points and smoking 3 points), with an area under the curve of 0.65 and a cut-off point of 2 . In the validation cohort the area under the curve was 0.66. Compared with PHQ 9, the negative predictive value was $84 \%$. Conclusions: Health workers in Argentina had a high prevalence of major depression during the pandemic, associated with predictive factors. A test was developed to detect those who are at low risk for it.

Keywords: Depression. Healthcare workers. SARS-CoV-2.

\section{Introducción}

Ante la expansión de la pandemia de enfermedad por coronavirus 2019 (COVID-19) y su llegada a la República Argentina, las autoridades sanitarias establecieron medidas para su contención y mitigación, dentro de las cuales el Aislamiento Social Preventivo y Obligatorio (ASPO) fue una de las principales ${ }^{1}$. Según datos internacionales y nuestra encuesta ImPPaCTS-SAC. $20^{3}$, el ASPO derivó en efectos psicológicos negativos como aumento de la ansiedad y depresión, la sensación de burnout, y de hábitos poco saludables como el sedentarismo y el consumo de alcohol o tabaco, tanto en la población general como en el personal de salud ${ }^{2-6}$. La depresión ha sido uno de los efectos psicofísicos más prevalentes, oscilando entre el 25 y el $33 \%$ de los trabajadores de la salud encuestados, según diferentes series realizadas durante esta y otras pandemias ${ }^{2-11}$.

El objetivo de este estudio es describir las diferentes variables dentro de la cohorte de trabajadores de la salud de Argentina incluidos en la encuesta ImPPaCTS-SAC.20 que se asocian al desarrollo de depresión mayor durante la pandemia por COVID-19, causada por el coronavirus 2 del síndrome respiratorio agudo grave (SARS-CoV-2), y generar un puntaje para su pesquisa.

\section{Material y métodos}

Subanálisis de la encuesta ImPPaCTS-SAC. $20^{2}$. Brevemente, fue una encuesta autoadministrada, anónima, realizada desde junio a septiembre de 2020, que incluyó trabajadores de la salud de Argentina. Se recolectaron datos socioeconómicos y demográficos, manejo del estrés y tres herramientas de pesquisa poblacional de burnout (Mini Z), ansiedad (GAD 7, Generalized Anxiety Disorder 7) y depresión (PHQ 9, Patient Health Questionnaire 9)2. La puntuación GAD 7 ha sido creada para la pesquisa de trastorno de ansiedad generalizada, está compuesto por 7 ítems y con un punto de corte de mayor o igual a 10 (la versión española tiene un coeficiente $\alpha$ de Cronbach de 0.93 , una sensibilidad del $86.8 \%$ y especificidad del
93.4\% ${ }^{12,13}$. La herramienta PHQ 9 establece el diagnóstico presuntivo de depresión mayor si hay por lo menos 5 ítems puntuados al menos como «más de la mitad de los días» o "casi todos los días», y al menos uno de los ítems positivos corresponde a las preguntas 102 , mientras que será depresión menor si hay por lo menos 2 a 4 ítems puntuados al menos como «más de la mitad de los días» o «casi todos los días», y al menos uno de los ítems positivos corresponde a las preguntas $1 \circ 2^{14-19}$. El puntaje mini $Z$ valora diferentes aspectos del ámbito laboral, y la pregunta número 3 detecta la sensación de burnout (sentirse mentalmente agotado ${ }^{20}$. En el estudio ImPPaCTS-SAC.20 se calcularon los coeficientes de confiabilidad ( $\alpha$ de Cronbach): GAD 7 ( $\alpha$ de Cronbach: 0,91), PHQ 9 ( $\alpha$ de Cronbach: 0,9) y cuestionario Mini $Z$ ( $\alpha$ de Cronbach: 0,728$)^{2}$. De la población con depresión mayor según el puntaje $\mathrm{PHQ}$ 9 , se evaluaron variables asociadas a esta y se las sometió a un análisis multivariante para detectar factores predictores.

\section{Análisis estadístico general}

Las variables cualitativas fueron expresadas como frecuencias absolutas y relativas, las cuantitativas como mediana e intervalo intercuartil (RIC) 25-75 o promedio y desvío estándar según su distribución. El análisis univariante de variables cualitativas fue realizado mediante el test de Chi cuadrada y las continuas mediante $U$ de Mann-Whitney o $t$ de Student según corresponda. Se asumió un error alfa de 0.05. El análisis multivariante se realizó mediante regresión logística.

\section{Construcción del puntaje de predicción}

Con las variables predictoras de depresión mayor en el análisis multivariante realizado en la cohorte completa, se realizó un nuevo modelo de regresión logística en una cohorte de derivación que fue constituida de manera aleatoria con el $50 \%$ de la muestra original. Al persistir significativas con las odd ratios (OR) se elaboró un puntaje. Se construyó una curva ROC (Receiver 
Tabla 1. Características basales de las poblaciones con y sin depresión mayor según el PHO 9 (Patient Health Questionnaire 9)

\begin{tabular}{|c|c|c|c|}
\hline & \multicolumn{2}{|c|}{ Depresión mayor } & \multirow[t]{2}{*}{$\mathbf{p}$} \\
\hline & $\begin{array}{c}\text { No } n=951 \\
(77.9 \%)\end{array}$ & $\begin{array}{c}\text { Sí } n=270 \\
(22.1 \%)\end{array}$ & \\
\hline Sexo femenino & $547(63.4 \%)$ & $188(74.6 \%)$ & 0.001 \\
\hline Edad & 43 (RIC: $26-60)$ & 41 (RIC: 24-58) & 0.027 \\
\hline $\begin{array}{l}\text { Trabajador } \\
\text { independiente }\end{array}$ & $574(60.4 \%)$ & $144(53.3 \%)$ & 0.038 \\
\hline Trabajar UCO/UTI & $185(19.5 \%)$ & $77(28.5 \%)$ & 0.001 \\
\hline $\begin{array}{l}\text { Sentirse } \\
\text { discriminado }\end{array}$ & $352(37 \%)$ & $141(52.2 \%)$ & 0.001 \\
\hline Actividad física & $245(25.8 \%)$ & $59(21.9 \%)$ & 0.190 \\
\hline Yoga & $73(7.7 \%)$ & $32(11.9 \%)$ & 0.031 \\
\hline Actividad religiosa & $65(6.8 \%)$ & $25(9.3 \%)$ & 0.178 \\
\hline Psicoterapia & $143(15 \%)$ & $58(21.5 \%)$ & 0.012 \\
\hline Actividad artística & $129(13.6 \%)$ & $26(9.6 \%)$ & 0.087 \\
\hline $\begin{array}{l}\text { Consumo de } \\
\text { ansiolíticos }\end{array}$ & $77(8.1 \%)$ & $61(22.6 \%)$ & 0.001 \\
\hline $\begin{array}{l}\text { Uso de } \\
\text { antidepresivos }\end{array}$ & $19(2 \%)$ & $17(6.3 \%)$ & 0.001 \\
\hline Fumar & $29(3 \%)$ & $21(78 \%)$ & 0.001 \\
\hline $\begin{array}{l}\text { Consumo de } \\
\text { alcohol }\end{array}$ & $58(6.1 \%)$ & $28(10.4 \%)$ & 0.015 \\
\hline
\end{tabular}

UCO: unidad coronaria; UTI: unidad de terapia intensiva; RIC: intervalo intercuartílico.

Operating Characteristic) y se determinó un punto de corte equilibrando sensibilidad y especificidad; se determinaron los valores predictivos negativos y positivos para toda la cohorte original.

\section{Validación del puntaje}

La cohorte de validación constó de los casos restantes. Se calculó el puntaje para cada uno de ellos y se construyó una nueva curva ROC para determinar la calidad del modelo. Finalmente se aplicó este modelo a la cohorte completa y se determinaron la sensibilidad, especificidad, valores predictivos y negativos.

\section{Resultados}

De los 1,221 encuestados de la cohorte global de la encuesta IMPPACTS-SAC20, hubo 270 casos (22.1\%)

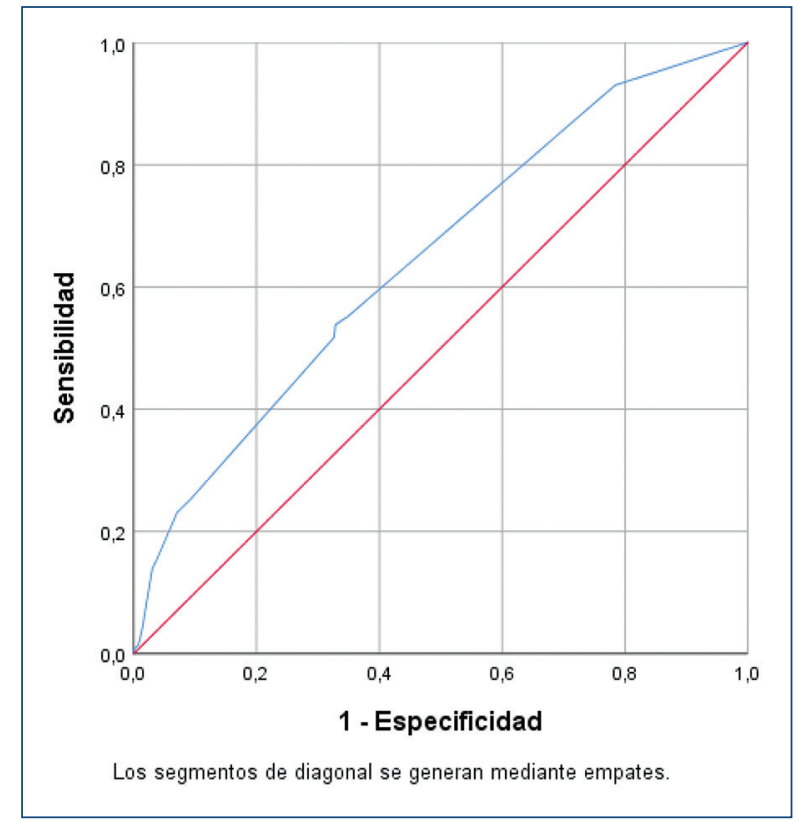

Figura 1. Área bajo la curva del modelo en la cohorte de derivación (área 0,65).

de depresión mayor según el PHQ 9. Las características basales de esta subpoblación y el resultado del análisis univariante pueden observarse en la tabla 1. No se observaron diferencias estadísticamente significativas en las tasas de depresión mayor detectadas en las diferentes regiones del país (Tabla 2). En el análisis multivariante, las variables asociadas con depresión mayor fueron sexo femenino (OR: 1.7; intervalo de confianza del 95\% [IC 95\%]: 1.2-2.3; $p=0.002$ ), sentirse discriminado (OR: 1.7; IC 95\%: 1.3-2.3; $p=0.001$ ), uso de ansiolíticos (OR: 3; IC 95\%: 1.9-4.6; $p=0.001$ ), tabaquismo (OR: 2.2; IC 95\%: 1.1-4.5; $p=0.025$ ) y trabajar en áreas de alta complejidad (OR: 1.6; IC 95\%: 1.1-2.3; $p=0.006$ ).

La cohorte de derivación constó de 569 encuestados (50\% de la muestra) y se excluyeron 55 por datos incompletos. Se elaboró un puntaje basado en las variables que se asociaron a depresión mayor en el análisis multivariante (sexo femenino 2 puntos, sentirse discriminado 1.5 puntos, uso de ansiolíticos 2.5 puntos y tabaquismo 3 puntos), con un área bajo la curva de 0.65 (IC 95\%: 60-70; p < 0.001) (Fig. 1), y se determinó un punto de corte de 2 equilibrando sensibilidad y especificidad. Con dicho punto de corte se establecieron los valores predictivos positivo y negativo correspondientes (Tabla 3). La cohorte de validación constó de 597 casos, de los cuales se excluyeron 51 por 
Tabla 2. Tasas de depresión mayor según el PHO 9 (Patient Health Questionnaire 9) entre diferentes regiones del país

\begin{tabular}{|l|c|c|}
\hline & Depresión mayor & p \\
\hline CABA y P.BsAs vs. resto & $199(23.1 \%)$ vs. $53(20.8 \%)$ & 0.440 \\
\hline CABA vs. resto & $131(22 \%)$ vs. $121(23.2 \%)$ & 0.629 \\
\hline P.BsAs vs. resto & $68(25.6 \%)$ vs. $184(21.6 \%)$ & 0.179 \\
\hline CABA vs. P.BsAs & $131(22 \%)$ vs. $121(23.2 \%)$ & 0.620 \\
\hline
\end{tabular}

CABA: Ciudad Autónoma de Buenos Aires; P.BsAs: Provincia de Buenos Aires.

Tabla 3. Sensibilidad, especificidad, valor predictivo positivo y negativo para el punto de corte obtenido (2 puntos) equilibrando sensibilidad y especificidad del modelo

\begin{tabular}{|l|c|c|c|c|}
\hline & \multicolumn{2}{|c|}{ PHO 9 } & \multirow{2}{*}{ Total } & \multirow{2}{*}{} \\
\cline { 2 - 4 } & $\begin{array}{c}\text { Depresión } \\
\text { Sí }\end{array}$ & $\begin{array}{c}\text { Depresión } \\
\text { No }\end{array}$ & & \\
\hline $\begin{array}{l}\text { Puntaje } \\
\text { positivo }\end{array}$ & 140 & 284 & 424 & VPP 33\% \\
\hline $\begin{array}{l}\text { Puntaje } \\
\text { negativo }\end{array}$ & 112 & 579 & 691 & VPN 87\% \\
\hline Total & 252 & 863 & 1,115 & \\
\hline & $\begin{array}{c}\text { Sensibilidad } \\
56 \%\end{array}$ & $\begin{array}{c}\text { Especificidad } \\
67 \%\end{array}$ & & \\
\hline
\end{tabular}

PHO 9: Patient Health Questionnaire 9; VPP: valor predictivo positivo; VPN: valor predictivo negativo.

Tabla 4. Sensibilidad, especificidad, valor predictivo positivo (VPP) y valor predictivo negativo (VPN) del modelo de predicción de depresión mayor

\begin{tabular}{|l|c|}
\hline & $\begin{array}{c}\text { Modelo de predicción de depresión mayor } \\
\text { Punto de corte 2 puntos }\end{array}$ \\
\hline Sensibilidad & $56 \%$ \\
\hline Especificidad & $67 \%$ \\
\hline VPP & $33 \%$ \\
\hline VPN & $84 \%$ \\
\hline
\end{tabular}

presentar datos incompletos, y utilizando el punto de corte mayor a 2 se obtuvo un área bajo la curva de 0.66 (IC 95\%: 0.62-0.70).

En la cohorte completa se tuvieron que excluir 106 casos por falta de datos, quedando una cohorte de 1,115 encuestados. Cuando se analizó el rédito diagnóstico comparado con la escala PHQ 9 (Tabla 4) se observó un valor predictivo negativo del $84 \%$, una likelihood ratio positiva y negativa de 1.69 y 0.66 , respectivamente.

\section{Discusión}

En nuestro estudio detectamos variables demográficas, socioeconómicas y de comportamiento frente al estrés relacionadas con el diagnóstico de depresión mayor en trabajadores de la salud en la República Argentina. Asimismo, en un contexto de alta prevalencia pudimos desarrollar un puntaje diagnóstico rápido y con variables fácilmente obtenibles que permitiría realizar una pesquisa de la población en riesgo.

En cuanto a la prevalencia de depresión en la población argentina durante la pandemia, un relevamiento argentino previo reportó en 1,059 personas durante el mes de junio síntomas de depresión en el $81 \%$ de los trabajadores de la salud encuestados ${ }^{21}$. Estos resultados son equiparables a nuestro trabajo, evidenciando elevados índices de síntomas que deben ponernos en alerta en términos de salud mental: $22.1 \%$ de depresión mayor, $10.9 \%$ de depresión menor y $4.5 \%$ con ideación suicida ${ }^{2}$. Por el contrario, en 2018 el Estudio epidemiológico de salud mental en población general de la República Argentina detectó síntomas de depresión mayor en el 8.7\% de los encuestados, lo que marca el aumento de este padecimiento como consecuencia de la pandemia ${ }^{22}$, justificado por la constante amenaza del posible contagio entre el personal de salud, el aislamiento social y familiar impuesto y la sobreexigencia laboral derivada. Asimismo, series internacionales apoyan estos hallazgos, con prevalencias de síntomas depresivos similares ${ }^{23-25}$.

El UK Household Longitudinal Study mostró que la prevalencia de malestar psicológico aumentó del 19.4\% en $2017-2019$ al $30.6 \%$ en abril de 2020 , siendo los grupos más severamente afectados las mujeres, jóvenes, asiáticos y personas con menor nivel de educación ${ }^{24}$. En el presente subanálisis, la presencia de depresión mayor se asoció independientemente con el sexo femenino, pero también con sentirse discriminado, el uso de ansiolíticos y el tabaquismo. En este sentido, en un metaanálisis de 115 artículos publicados hasta abril de 2020 que incluyó a 60,458 trabajadores de la salud asistiendo a pacientes con SARS/síndrome respiratorio de Oriente Medio/COVID-19, el 26.3\% presentaba depresión ${ }^{25}$.

En nuestra cohorte se detectó que el $13.4 \%$ del personal encuestado era tabaquista activo previo al ASPO, aunque aumentaron su consumo tras el mismo el $9.4 \%$, 
mientras que el 1.2\% inició dicho hábito, según ha sido publicado previamente ${ }^{2}$. Asimismo, otras estrategias no saludables para el control del estrés fueron el uso de ansiolíticos en el $11.3 \%^{2}$. Estos últimos han sido identificados como predictores claros de depresión en el presente estudio, y claramente son factores de riesgo que deben abordarse en el personal de salud, desde lo individual y lo colectivo. Si bien existen múltiples reportes que informan un aumento de estos hábitos no saludables durante la pandemia, recordemos que en este caso se trata de la subpoblación de trabajadores de la salud, por lo que las comparaciones con series nacionales e internacionales en la población general no pueden realizarse $6,7,25,26$.

Es importante destacar que el diagnóstico de depresión requiere un abordaje profundo por especialistas en la materia, por lo que ningún cuestionario puede suplantar el criterio clínico y los múltiples encuentros que muchas veces se requiere para abordar esta problemática. Sin embargo, a la hora de efectuar una pesquisa poblacional para detectar en la población general esta afección, existen herramientas autoadministradas 0 heteroadministradas que permiten identificar posibles casos de depresión para un posterior abordaje más profundo de estos ${ }^{12-20}$. Este es el caso del PHQ 9, el cual está ampliamente validado con buena sensibilidad y especificidad para la pesquisa comunitaria de depresión ${ }^{14-19}$. Nuestro trabajo ha detectado cuatro factores predictores de depresión mayor en trabajadores de la salud durante la pandemia de COVID-19, que nos permitió construir un puntaje para la pesquisa de esta afección en esta subpoblación. Cabe destacar que este nuevo puntaje presenta una baja sensibilidad y valor predictivo positivo, pero alto valor predictivo negativo, en comparación con el PHQ 9, por lo que es útil para descartar a aquellos trabajadores con baja probabilidad de desarrollar depresión mayor en la actual situación epidemiológica. En aquellos trabajadores de la salud que presenten este puntaje positivo, sostenemos que deberían abordarse con cuestionarios de mayor sensibilidad y eventualmente la consulta con el especialista.

La depresión y otros trastornos mentales en trabajadores de salud como consecuencia de la actual pandemia sin duda comprometen la calidad de atención médica de los pacientes, aumentan los costos de los sistemas de salud devenido del ausentismo laboral y, principalmente, conllevan un empeoramiento franco de la salud no solo del individuo afectado, sino también de sus familias ${ }^{27}$. En este sentido, resulta fundamental pesquisar tempranamente síntomas de depresión, ansiedad o burnout en los trabajadores de la salud por medio de equipos multidisciplinarios con apoyo psicológico y social en los centros de salud a fin de evitar consecuencias severas laborales, familiares y en la salud biopsicosocial de cada trabajador de la salud de Argentina durante la pandemia de COVID-19 y a futuro.

Nuestro estudio es observacional en naturaleza y presenta los sesgos inherentes a este diseño. No contamos cohorte externa para validar nuestro puntaje diagnóstico y su generalización en la población no médica o médica que vive situaciones sociales diferentes a las locales probablemente sea limitada. No hemos validado nuestro modelo de manera prospectiva.

\section{Conclusión}

En la población de trabajadores de la salud de Argentina durante la pandemia de COVID-19 hemos detectado una alta prevalencia de depresión mayor. Encontramos variables con fuerte asociación a este diagnóstico por medio de las cuales pudimos elaborar un puntaje diagnóstico con alto valor predictivo negativo para detectar a quienes tengan bajo riesgo de padecerla.

\section{Agradecimientos}

Al Consejo de insuficiencia cardiaca e hipertensión pulmonar y al Consejo de aspectos psicosociales de la Sociedad Argentina de Cardiología (SAC).

\section{Financiamiento}

La presente investigación no ha recibido ninguna beca específica de agencias de los sectores públicos, comercial o sin ánimo de lucro.

\section{Conflicto de intereses}

Los autores declaran no tener conflicto de intereses.

\section{Responsabilidades éticas}

Protección de personas y animales. Los autores declaran que para esta investigación no se han realizado experimentos en seres humanos ni en animales.

Confidencialidad de los datos. Los autores declaran que han seguido los protocolos de su centro de trabajo sobre la publicación de datos de pacientes.

Derecho a la privacidad y consentimiento informado. Los autores han obtenido el consentimiento informado de los pacientes y/o sujetos referidos en el artículo. 


\section{Bibliografía}

1. Organización Mundial de la Salud. COVID-19: Cronología de la actuación de la OMS [Internet]. Organización Mundial de la Salud; 27 de abril de 2020 [consultado: Octubre de 2020]. Disponible en https://www.who.int/ es/news-room/detail/27-04-2020-who-timeline---covid-19

2. Scatularo CE, Battioni L, Bellia S, Costa de Robert S, Gatti M, Racki M et al. Impacto psicofísico de la pandemia COVID-19 en trabajadores de la salud en Argentina: Encuesta ImPPaCTS-SAC.20. Rev Argent Cardiol. 2021;89:196-202.

3. Wang C, Pan R, Wan X, Tan Y, Xu L, Ho CS et al. Immediate psychological responses and associated factors during the initial stage of the 2019 coronavirus disease (COVID-19) epidemic among the general population in China. Int J Environ Res Public Health. 2020;17:17-29.

4. Sandín B, Valiente RM, García-Escalera J, Chorot P. Impacto psicológico de la pandemia de COVID-19: Efectos negativos y positivos en la población española asociados al período de confinamiento nacional. Rev Psicopatol Psicol Clin. 2020;25(1):1-22.

5. Vanderbruggen N, Matthys F, van Laere S, Zeeuws D, Santermans L, van den Ameele $S$ et al. Self-reported alcohol, tobacco, and cannabis use during COVID-19 lockdown measures: Results from a web-based survey. Eur Addict Res. 2020;26(6):309-15.

6. Australian Institute of Health and Welfare. Alcohol, tobacco \& other drugs in Australia [Internet]. Canberra: Australian Institute of Health and Welfare [consultado: 2 de Noviembre de 2020]. Disponible en: Https://www. aihw.gov.au/reports/alcohol/alcohol-tobacco-other-drugs-australia

7. Tomlin J, Dalgleish-Warburton B, Lamph G. Psychosocial support for healthcare workers during the COVID-19 pandemic. Front Psychol. 2020;11:1960.

8. Cai H, Tu B, Ma J, Chen L, Fu L, Jiang Y et al. Psychological impact and coping strategies of frontline medical staff in Hunan between January and March 2020 during the outbreak of coronavirus disease 2019 (COVID-19) in Hubei, China. Med Sci Monit. 2020;26:E924171.

9. Bozdag F, Ergün N. Psychological resilience of healthcare professionals during COVID-19 pandemic. Psychol Rep. 2021:124(6):2567-86.

10. Setiawati $Y$, Wahyuhadi J, Joestandari F, Maramis MM, Atika A. Anxiety and resilience of healthcare workers during COVID-19 pandemic in Indonesia. J Multidiscip Healthc. 2021;14:1-8

11. Vagni M, Maiorano T, Giostra V, Pajardi D. Coping with COVID-19: Emergency stress, secondary trauma and self-efficacy in healthcare and emergency workers in Italy. Front Psychol. 2020;11:566912.

12. Spitzer RL, Kroenke K, Williams JBW, Löwe B. A brief measure for assessing generalized anxiety disorder. Arch Intern Med. 2006;166:1092-97.

13. Garcia-Campayo J, Zamorano E, Ruiz MA, Pardo A, Perez-Paramo M Lopez-Gomez V et al. Cultural adaptation into Spanish of the generalized anxiety disorder-7 (GAD-7) scale as a screening tool. Health Qual Life Outcomes. 2010;8(1):8.
14. Spitzer R, Kroenke K, Williams J. Validation and utility of self-report version of PRIME-ME: The PHQ primary care study. J Am Med Assoc. 1999;282:1737-44.

15. Kroenke K, Spitzer R, Williams J. The PHQ-9 validity of a brief depression severity measure. J Gen Intern Med. 2001;16:606-13.

16. Löwe B, Spitzer R, Gräfe K, Kroenke K, Quenter A, Zipfel S et al. Comparative validity of three screening questionnaires for DSM-IV depressive disorders and physicians diagnoses. J Affect Disord. 2004;78:131-40.

17. Martin A, Rief W, Klaiberg A, Braehler E. Validity of the Brief Patient Health Questionnaire Mood Scale (PHQ-9) in the general population. Gen Hosp Psychiatry. 2006;28:71-7.

18. Backenstrass M, Frank A, Joest K, Hingmann S, Mundt Ch, Kronmüller KT. A comparative study of non specific depressive symptoms and minor depression regarding functional impairment and associated characteristics in primary care. Compr Psychiatry. 2006;47:35-41.

19. Diez-Quevedo C, Rangil T, Sánchez-Planell L, Kroenke K, Spitzer R. Validation and Utility of the Patient Health Questionnaire in Diagnosing Mental Disorders in 1003 General Hospital Spanish Inpatients. Psychosom Med. 2001;63:679-86.

20. Rohland BM, Kruse GR, Rohrer JE. Validation of a single-item measure of burnout against the Maslach Burnout Inventory among physicians. Stress Health. 2004;20(2):75-9.

21. Giardino DL, Huck-Iriart C, Riddick M. The endless quarantine: The impact of the COVID-19 outbreak on healthcare workers after three months of mandatory social isolation in Argentina. Sleep Medicine. 2020;76:16-25.

22. Stagnaro JC, Cía A, Vázquez N, Vommaro H, NemirovskyM, Serfaty E et al. Estudio epidemiológico de salud mental en población general de la República Argentina. Rev Arg Psiquiat. 2018;29:275-99.

23. Figueroa R. El impacto psicológico de la pandemia de COVID-19 en el personal de salud: Un panorama preocupante. ARS MEDICA Revista de Ciencias Médicas. 2020;45(3):3-5.

24. Niedzwiedz CL, Green MJ, Benzeval M, Campbell D, Craig P, Demou E et al. Mental health and health behaviours before and during the initial phase of the COVID-19 lockdown: Longitudinal analyses of the UK Household Longitudinal Study. J Epidemiol Community Health. 2021;75(3):224-31.

25. Salazar de Pablo G, Vaquerizo-Serrano J, Catalan A, Arango C, Moreno $\mathrm{C}$, Ferre $\mathrm{F}$ et al. Impact of coronavirus syndromes on physical and mental health of health care workers: Systematic review and meta-analysis. J Affect Disord. 2020;275:48-57.

26. Falkingham J, Evandrou M, Qin M, Vlachantoniet A. Prospective longitudinal study of 'Sleepless in Lockdown': Unpacking differences in sleep loss during the coronavirus pandemic in the UK. BMJ Open. 2022;12(1):E053094

27. Rodziewicz TL, Houseman B, Hipskind JE. Medical error reduction and prevention [Internet]. StatPearls. Treasure Island (FL): StatPearls Publishing; 4 de enero de 2021. Disponible en: Https://www.ncbi.nlm.nih. gov/books/NBK499956 